\title{
PERFIL DA ASSISTÊNCIA AO PARTO NORMAL EM UMA MATERNIDADE ESCOLA FEDERAL
}

\section{NATURAL CHILDBIRTH CARE PROFILE AT A MATERNITY HOSPITAL OF A FEDERAL SCHOOL}

\section{PERFIL DE LA ASISTENCIA AL PARTO NORMAL EN UNA MATERNIDAD ESCUELA FEDERAL}

\section{Francini de Souza Rodrigues ${ }^{1}$, Cristiane Barbosa Batista ${ }^{2}$, Geiza Martins Barros ${ }^{3}$}

Como citar esse artigo: Rodrigues FS, Batista CB, Barros GM. Perfil da assistência ao parto normal em uma maternidade escola federal. Rev Enferm Atenção Saúde [Internet]. 2021 [acesso em: ]; 10(3):e202131. doi: https://doi.org/ 10.18554/reas.v10i3.4567

\section{RESUMO}

Objetivo: Caracterizar a assistência ao parto normal em uma Maternidade Escola Federal, no município do Rio de Janeiro. Método: Pesquisa descritiva, de abordagem quantitativa. Os dados foram coletados a partir dos registros de partos normais locais no período de julho a dezembro de 2018. No total foram realizados 466 partos, sendo 97 excluídos pelos critérios estabelecidos, dessa forma, foram analisados 369 registros. Para a análise dos dados foi utilizado o programa Microsoft Excel 2013 com a descrição dos dados absolutos e relativos. Resultados: a assistência ao parto normal contou com práticas baseadas em evidências científicas, como a garantia do acompanhante no trabalho de parto e parto, com 90,79\% e estímulo ao aleitamento materno, em 78,32\%, mas também, fez-se presente práticas desaconselhadas rotineiramente tais como: amniotomia, em 30,08\% e episiotomia em 20,87\%. Conclusão: observou-se que a assistência na referida maternidade se baseou, predominantemente, nas boas práticas assistenciais.

Descritores: Parto Normal; Parto Humanizado; Tocologia.

\footnotetext{
${ }_{1}^{1}$ Enfermeira. Especialista em Saúde Perinatal pela Maternidade Escola da UFRJ. Rio de Janeiro, Brasil. Email: francinidesouza.rodrigues@gmail.com

2 Mestre em Epidemiologia em Saúde Pública na ENSP/FIOCRUZ. Especialista em Saúde da Família pela Escola Nacional de Saúde Pública Sérgio Arouca - FIOCRUZ. Graduada em Enfermagem pela Universidade Federal do Estado do Rio de Janeiro. E-mail: cristiane.batist@gmail.com

3 Doutoranda em Ciências da Saúde (IFF/Fiocruz). Mestre em Ciências da Saúde (IFF/Fiocruz). Enfermeira Obstétrica (UERJ).E-mail: geiza_mb@hotmail.com
} 


\begin{abstract}
ABSTRACT
Objective: This study aimed to characterize the care to natural childbirth at the maternity hospital of a federal school located in the city of Rio de Janeiro. Method: This is a quantitative descriptive study. Data were collected from the records of local natural childbirths from July to December 2018. In total, 466 births were performed, 97 of which were excluded according to the inclusion criteria; then 369 records were analyzed. Data were analyzed using Microsoft Excel 2013 with the description of absolute and relative data. Results: Care to natural childbirth was provided with evidence-based practices, including the presence of a companion during labor and delivery in $90.79 \%$ of the cases, and encouraging breastfeeding in $78.32 \%$. Practices that are not usually recommended were also observed, like amniotomy in $30.08 \%$ and episiotomy in $20.87 \%$. Conclusion: Care in the studied maternity was predominantly based on good care practices.
\end{abstract}

Descriptors: Natural Childbirth; Humanizing Delivery; Midwifery.

\title{
RESUMEN
}

Objetivo: Caracterizar la asistencia al parto normal en una Maternidad Escuela Federal, en la ciudad de Rio de Janeiro. Método: Investigación descriptiva, con enfoque cuantitativo. Se recolectaron datos de los registros de partos normales locales de julio a diciembre de 2018. En total se realizaron 466 partos, de los cuales 97 fueron excluidos por los criterios establecidos, por lo que se analizaron 369 registros. Para el análisis de los datos se utilizó Microsoft Excel 2013 con la descripción de datos absolutos y relativos. Resultados: En la asistencia al parto normal se realizaron prácticas basadas en evidencia científica, como garantizar un acompañante en el trabajo de parto y parto en el 90,79\% de los casos y fomentar la lactancia materna en el 78,32\%, pero también se realizaron prácticas que no se aconsejan habitualmente como: amniotomía en el 30,08\% y episiotomía en el 20,87\%. Conclusión: Se observó que la asistencia en la maternidad referida se basó predominantemente en buenas prácticas de cuidado.

Descriptores: Parto Normal; Parto Humanizado; Tocología.

\section{INTRODUÇÃO}

O processo do nascimento sofreu modificações ao longo dos anos, onde inicialmente, o parto era realizado, em sua maior parte, nos domicílios das parturientes e, posteriormente, passa a se realizar em ambientes hospitalares e com uso das mais diversas tecnologias de assistência à saúde. ${ }^{1} \quad$ Frente a essas mudanças, na segunda metade do século $\mathrm{XX}$, iniciou-se mundialmente um movimento de retorno às origens do parto, onde a humanização da assistência ao parto voltou a ser defendida, trazendo de volta o Rev Enferm Atenção Saúde [Online]. Out/Dez 2021; 10(3):e202131 protagonismo da mulher durante o parto normal. Tal defesa se materializou em documentos e políticas públicas que possibilitam novas práticas durante a assistência ao parto, baseadas em evidências científicas.

Deste modo, em 14 de fevereiro de 2017 foi publicada a portaria 353, do Ministério da Saúde, que aponta diretrizes, baseadas em evidências científicas, para a assistência ao parto normal em mulheres com gestação de risco habitual. As recomendações resultaram de um árduo estudo sobre trabalhos científicos de 
diferentes qualidades, sendo considerados principalmente aqueles mais robustos e confiáveis. ${ }^{2}$

Dentre as práticas que devem ser encorajadas estão a utilização do partograma, oferta de líquidos via oral ou dieta leve durante o trabalho de parto e parto, métodos não invasivos e não farmacológicos para alívio da dor, livre movimentação e liberdade de posição da mulher durante o trabalho de parto. A analgesia deve ser uma opção à mulher, após tentativas com os métodos não farmacológicos para alívio de dor. $\mathrm{O}$ contato pele a pele entre mãe e recém-nato e amamentação na primeira hora de vida são essenciais para a manutenção do vínculo mãe-bebê e auxilia na redução da hemorragia pós-parto. ${ }^{3}$

Em relação às práticas claramente prejudiciais ou ineficazes e que devem ser eliminadas encontram-se uso rotineiro de enema e tricotomia. A episiotomia de rotina bem como a manobra de Kristeller estão desaconselhados. Há também a orientação de se evitar puxos dirigidos.$^{3}$

A amniotomia precoce, o clampeamento precoce do cordão umbilical e a administração de ocitocina após a analgesia não devem ser utilizados de maneira rotineira e devem ser utilizados apenas nos casos necessários. A tração controlada do cordão umbilical do terceiro estágio do trabalho de parto pode auxiliar Rev Enferm Atenção Saúde [Online]. Out/Dez 2021; 10(3):e202131 na redução do risco de remoção manual da placenta, podendo ser usada nas mulheres que optarem por isso. ${ }^{3}$

Dentre as mais recentes iniciativas referentes ao parto humanizado, no Brasil, pode-se destacar, o projeto "Apice On" em execução em 97 hospitais de ensino no território nacional que propõe "qualificar nos campos de atenção/cuidado ao parto e nascimento, planejamento reprodutivo pósparto e pós-aborto, atenção às mulheres em situações de violência sexual e de abortamento e aborto legal". ${ }_{4}$

Em 2018, a Organização Mundial de Saúde (OMS) pública a "Intrapartum care for a positive childbirth experience", um importante documento que traz 56 recomendações científicas para o trabalho de parto, parto e pós-parto, assim como também elenca uma série de cuidados ao recém-nascido, com o objetivo de tornar a experiência do parto normal humanizada. ${ }^{5}$

Diante às recomendações oficiais descritas como boas práticas de atenção e por ser a unidade do estudo (Maternidade Escola) formadora de profissionais especialistas na área, objetivou-se com o estudo: Caracterizar a assistência ao parto normal em uma Maternidade Escola Federal, no município do Rio de Janeiro. 


\section{MÉTODO}

Trata-se de um estudo descritivo, transversal e de abordagem quantitativa. $\mathrm{O}$ cenário do estudo foi em uma Maternidade Escola Federal, localizada no município do Rio de Janeiro, pública, especializada, que dispõe de assistência ambulatorial e hospitalar, multiprofissional, oferecendo linhas de cuidado específicas na atenção à saúde de gestantes e recém-nascidos de alto risco. Possui ambulatórios especializados na assistência pré-natal (hipertensão arterial, diabetes, gestação gemelar, patologias fetais e adolescentes), programa de rastreio de risco para gestantes no primeiro trimestre, planejamento familiar para mulheres de risco, genética pré-natal e medicina fetal.

Os dados foram coletados a partir de um livro de registros de parto normal da referida instituição, sendo a coleta guiada por um instrumento de coleta de dados elaborado pelas autoras. Esse instrumento continha afirmações fechadas referentes as práticas adotadas na assistência ao parto normal preenchidas com sim e não. A coleta de dados ocorreu no período de junho a agosto de 2019.

Os critérios de inclusão da pesquisa foram os registros das mulheres que tiveram partos normais realizados no centro obstétrico da instituição e os critérios de exclusão foram os registros das Rev Enferm Atenção Saúde [Online]. Out/Dez 2021; 10(3):e202131 mulheres que também tiveram parto normal, mas que deram entrada em período expulsivo; que tiveram partos classificados como pré-termo, definidos como idade gestacional inferior de 37 semanas; que não continham idade gestacional registrada e os partos realizados fora do centro obstétrico. Os partos gemelares foram considerados somente como um parto, sendo aplicados os mesmos critérios, tanto de exclusão quanto de inclusão. Foram analisados todos os registros de julho a dezembro de 2018, que contou com 466 partos. Destes, 97 partos foram excluídos de acordo com os critérios estabelecidos. Sendo assim, 369 partos foram elegíveis para o presente estudo.

Para a execução deste estudo foram respeitados os aspectos éticos em pesquisa envolvendo seres humanos pela resolução 466/12 do Conselho Nacional de Saúde. ${ }^{6}$ A fim de ressaltar a importância da confidencialidade e os aspectos éticos em pesquisa, o presente estudo foi encaminhado ao Comitê de Ética e Pesquisa, sendo aprovado, com número de parecer 3.339.596.

Para a análise de dados foi utilizado o programa Microsoft Excel 2013. O programa foi utilizado para a condensação dos dados após a coleta e também para posterior realização da estatística descritiva, com discriminação das 
frequências absolutas e relativas, sendo apresentados em tabelas.

Depois de coletadas, as variáveis estudadas foram agrupadas em categorias, sendo elas: $1 . \quad$ Características sociodemográficas e histórico obstétrico (raça/cor, faixa etária, realização do prénatal e número de partos); 2. Práticas de assistência ao parto normal baseadas em evidências científicas (presença de acompanhante no trabalho de parto e parto, liberação de dieta, utilização de métodos não farmacológicos para o alívio da dor, analgesia medicamentosa, clampeamento oportuno do cordão umbilical, contato pele a pele e amamentação na primeira hora de vida) e 3. Práticas de assistência ao parto normal desaconselhadas rotineiramente (ocitocina artificial no trabalho de parto, amniotomia, episiotomia e posição litotômica) e prática proscrita (manobra de Kristeller).

\section{RESULTADOS}

$\mathrm{Na}$ tabela 1, referente às características sociodemográficas e histórico obstétrico, ficou demonstrado que a raça/cor autodeclarada de maior predominância foi a parda, seguida pela branca e negra. Em relação à faixa etária observou-se que a predominância foi entre 20 a 35 anos.

No que diz respeito ao pré-natal, em quase sua totalidade de mulheres o realizou. Quanto a paridade, pouco menos da metade das mulheres eram nulíparas. 
Tabela 1 - Distribuição de variáveis das características sociodemográficas e histórico obstétrico no período de julho a dezembro de 2018.

\begin{tabular}{|c|c|c|c|}
\hline \multirow[t]{2}{*}{ 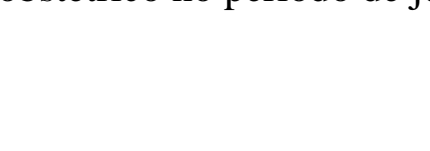 } & \multicolumn{3}{|l|}{ Raça/Cor } \\
\hline & & $\mathbf{N}$ & $\%$ \\
\hline & Branca & 98 & 26,56 \\
\hline & Negra & 63 & 17,07 \\
\hline & Parda & 181 & 49,05 \\
\hline & Amarela & 1 & 0,27 \\
\hline & Indígena & 1 & 0,27 \\
\hline & Sem Informação & 25 & 6,78 \\
\hline & \multicolumn{3}{|c|}{ Faixa etária } \\
\hline & & $\mathbf{N}$ & $\%$ \\
\hline & Entre 15 e $<20$ anos & 39 & 10,57 \\
\hline & Entre 20 e 35 anos & 264 & 71,54 \\
\hline & Maior que 35 anos & 51 & 13,82 \\
\hline & Sem Informação & 15 & 4,07 \\
\hline & \multicolumn{3}{|c|}{ Pré natal } \\
\hline & & $\mathbf{N}$ & $\%$ \\
\hline & Sim & 363 & 98,37 \\
\hline & Não & 2 & 0,54 \\
\hline & Sem Informação & 4 & 1,09 \\
\hline & \multicolumn{3}{|c|}{ Paridade } \\
\hline & & $\mathbf{N}$ & $\%$ \\
\hline & Nulíparas & 172 & 46,61 \\
\hline & 1 parto & 124 & 33,60 \\
\hline & $\geq$ a 2 partos & 72 & 19,51 \\
\hline & Sem Informação & 1 & 0,27 \\
\hline
\end{tabular}

Fonte: Livro de registro de parto normal, 2019. 
A tabela 2 apresenta algumas das práticas baseadas em evidências científicas preconizadas para o parto normal. Diante das informações desta tabela, podemos afirmar que a maioria das mulheres esteve acompanhada durante o trabalho de parto e do parto, assim como foi fornecida a devida alimentação neste período. Além disso, pouco mais da metade das mulheres fizeram uso do banho de aspersão como método não farmacológico para alívio da dor. Observou-se que em pouco mais da metade dos partos não foi requerido o uso de analgesia medicamentosa.

Diante às práticas relacionadas ao recém-nascido, identificou-se que em mais da metade dos partos houve o clampeamento oportuno do cordão, assim como o contato pele a pele imediato e o aleitamento materno.

Tabela 2 - Distribuição de variáveis das práticas de assistência ao parto normal com base nas evidências científicas no período de julho a dezembro de 2018.

\begin{tabular}{|c|c|c|}
\hline \multicolumn{3}{|c|}{ Acompanhante } \\
\hline & $\mathbf{N}$ & $\%$ \\
\hline Sim & 335 & 90,79 \\
\hline Não & 34 & 9,21 \\
\hline \multicolumn{3}{|l|}{ Dieta } \\
\hline & $\mathbf{N}$ & $\%$ \\
\hline Zero & 29 & 7,86 \\
\hline Sim & 332 & 89,97 \\
\hline Sem Informação & 8 & 2,17 \\
\hline \multicolumn{3}{|c|}{ Métodos não farmacológicos para alívio da dor: } \\
\hline & $\mathbf{N}$ & $\%$ \\
\hline Banho & 242 & 65,58 \\
\hline Exercícios respiratórios & 176 & 47,70 \\
\hline Massagem & 140 & 37,94 \\
\hline Deambulação & 173 & 46,88 \\
\hline Movimentos Facilitadores & 131 & 35,50 \\
\hline Bola & 113 & 30,62 \\
\hline Música & 60 & 16,26 \\
\hline Outros & 23 & 6,30 \\
\hline Não aceitou & 11 & 2,98 \\
\hline Sem Informação / Não realizado & 72 & 19,51 \\
\hline \multicolumn{3}{|l|}{ Analgesia } \\
\hline & $\mathbf{N}$ & $\%$ \\
\hline Sim & 147 & 39,84 \\
\hline Não & 221 & 59,89 \\
\hline Sem Informação & 1 & 0,27 \\
\hline
\end{tabular}




\begin{tabular}{|c|c|c|}
\hline \multicolumn{3}{|c|}{ Clampeamento oportuno do cordão umbilical } \\
\hline & $\mathbf{N}$ & $\%$ \\
\hline Sim & 296 & 80,22 \\
\hline Não & 68 & 18,43 \\
\hline Sem Informação & 5 & 1,35 \\
\hline \multicolumn{3}{|c|}{ Contato pele a pele } \\
\hline & $\mathbf{N}$ & $\%$ \\
\hline Imediato & 312 & 84,55 \\
\hline Primeiros 30 minutos & 24 & 6,50 \\
\hline Primeira hora & 2 & 0,54 \\
\hline Não houve & 29 & 7,87 \\
\hline Sem Informação & 2 & 0,54 \\
\hline \multicolumn{3}{|c|}{ Aleitamento materno } \\
\hline & $\mathbf{N}$ & $\%$ \\
\hline Sim & 289 & 78,32 \\
\hline Não & 72 & 19,51 \\
\hline Sem Informação & 8 & 2,17 \\
\hline
\end{tabular}

* Não soma $100 \%$ na porcentagem devido à utilização de mais de um método.

Fonte: Livro de registro de parto normal, 2019.

Na tabela 3 podemos observar o quantitativo de mulheres submetidas às práticas de assistência ao parto normal desaconselhadas de forma rotineira e à prática proscrita pelas evidências científicas atuais. Em relação às práticas de assistência ao parto normal desaconselhadas de forma rotineira observaram-se que pouco mais da metade dos partos houve o uso de ocitocina artificial, amniotomia e episiotomia foram utilizadas de forma menos expressiva, porém a posição litotômica obteve número bastante significativo. $\mathrm{Na}$ prática de assistência ao parto normal proscrita observou-se a manobra de Kristeller sendo utilizada. 
Tabela 3 - Distribuição de variáveis das práticas de assistência ao parto normal desaconselhadas rotineiramente e prática proscrita no período de julho a dezembro de 2018.

\begin{tabular}{|c|c|c|}
\hline \multicolumn{3}{|c|}{ Ocitocina no trabalho de parto } \\
\hline & $\mathbf{N}$ & $\%$ \\
\hline $\operatorname{Sim}$ & 202 & 54,74 \\
\hline Não & 167 & 45,26 \\
\hline \multicolumn{3}{|c|}{ Amniotomia } \\
\hline & $\mathbf{N}$ & $\%$ \\
\hline Sim & 111 & 30,08 \\
\hline Não & 251 & 68,02 \\
\hline Sem Informação & 7 & 1,90 \\
\hline \multicolumn{3}{|c|}{ Episiotomia } \\
\hline & $\mathbf{N}$ & $\%$ \\
\hline Sim & 77 & 20,87 \\
\hline Não & 291 & 78,86 \\
\hline Sem Informação & 1 & 0,27 \\
\hline \multicolumn{3}{|c|}{ Posição de nascimento } \\
\hline & $\mathbf{N}$ & $\%$ \\
\hline Litotômica & 321 & 86,99 \\
\hline Não litotômica & 48 & 13,01 \\
\hline
\end{tabular}

\begin{tabular}{c|c|c}
\hline \multicolumn{3}{|c}{ Realização de kristeller } \\
\hline & $\mathbf{N}$ & $\mathbf{\%}$ \\
\hline Sim & 11 & 2,98 \\
\hline Não & 356 & 96,48 \\
\hline Sem Informação & 2 & 0,54 \\
\hline
\end{tabular}

Fonte: Livro de registro de parto normal, 2019.

\section{DISCUSSÃO}

O perfil sociodemográfico do estudo evidenciou que $49,05 \%$ da população se autodeclarou de cor parda. Estudo que avaliou o perfil obstétrico e neonatal de puérperas atendidas em duas maternidades públicas de São Paulo apontou percentual semelhante, com $55,2 \%$ das puérperas autodeclaradas como pardas. $^{7}$
O estudo apresenta taxa de 71,54\% para idade entre 20 a 35 anos. Dados estes semelhantes ao estudo que avaliou o modelo de maternidade respeitosa em São Paulo, com 69,8\%. ${ }^{8}$ Tais dados podem apontar para um envelhecimento da população estudada, demonstrando que menos mulheres se tornaram jovens mães.

Em relação ao pré-natal, é possível observar que $98,37 \%$ das mulheres o realizaram durante a gestação atual neste estudo. Esse resultado vai ao encontro de 
outro, que apresenta praticamente a mesma porcentagem, com $98,3 \%$ entre as puérperas. $^{7}$ Tal situação aponta para a acessibilidade às consultas pré-natais.

Os achados em relação aos índices da paridade para este estudo apontam taxas de $46,61 \%$ para nulíparas, $33,60 \%$ para um parto anterior e $19,51 \%$ para dois ou mais partos anteriores. É interessante ressaltar que estes dados são diferentes dos encontrados no estudo que avaliou a atenção ao parto e nascimento em um hospital universitário na cidade de Porto Alegre, Rio Grande do Sul, comparando as práticas desenvolvidas após a implantação da rede cegonha entre os anos de 2012 e 2016, onde o mesmo obteve no ano de 2016 como resultado: 59\% para nulíparas, $24,8 \%$ para um parto anterior e $16,2 \%$ para dois ou mais partos anteriores. ${ }^{9}$

Quando analisada a presença de um acompanhante durante o trabalho de parto, o estudo aponta a taxa desta prática de $90,79 \%$. Dado este um pouco mais baixo do achado que apresentou 96,9\% em 2016 para a presença do acompanhante no trabalho de parto. ${ }^{9}$ É importante destacar que a Lei Federal $n^{\circ} 11.108$, de 07 de abril de 2005 prevê "garantir às parturientes o direito à presença de acompanhante durante o trabalho de parto, parto e pósparto imediato, no âmbito do Sistema Único de Saúde - SUS”. ${ }^{10}$ Pode-se assim notar que, na instituição estudada, essa lei é garantida, permitindo um acompanhante durante todo o período citado anteriormente, independente do sexo e de livre escolha da gestante.

A oferta de dieta liquida e alimentação no trabalho de parto é uma conduta recomendada pela OMS. ${ }^{5}$ Desse modo, o presente estudo aponta para uma taxa de oferta de dieta no trabalho de parto de $89,97 \%$. Este dado está acima do apresentado da pesquisa intitulada "Nascer no Brasil”, realizada no período de 2011 a 2012, que avaliou a assistência obstétrica em 266 hospitais espalhados em 191 municípios, porém, o artigo em questão analisado, avaliou o risco obstétrico habitual e apresentou $25,2 \%$ de taxa de alimentação no trabalho de parto para todas as mulheres. ${ }^{11}$

O uso dos métodos não farmacológicos para alívio da dor estão previstos e devem ser oferecidos à mulher em seu trabalho de parto, como recomenda o Ministério da Saúde em sua publicação sobre as diretrizes de assistência ao parto normal. $^{3}$ Vale ressaltar que, na maternidade estudada, os métodos não farmacológicos são oferecidos e compreendem a base dos cuidados da equipe de enfermagem no centro obstétrico, que inclusive consta com instrumentos padronizados para registro. Desta forma, neste estudo, 65,58\% das mulheres utilizaram banho, sendo seguidos 
de exercícios respiratórios, deambulação e massagem, com percentuais de 47,70\%, $46,88 \%$ e $37,94 \%$, respectivamente. Estudo feito sobre a assistência ao parto normal realizada pelas residentes de enfermagem obstétrica em uma maternidade pública municipal de grande porte do Rio de Janeiro encontrou como resultado para métodos empregados: $87,1 \%$ para técnicas de respiração, 50,7\% para deambulação, $44,9 \%$ para banho morno e $33,8 \%$ para massagem. ${ }^{12}$ Tais dados apontam que cada mulher possui suas particularidades e suas preferências no momento da escolha dos métodos não farmacológicos que deseja utilizar.

O uso dos métodos farmacológicos para alívio da dor, devem ser oferecidos após o emprego dos métodos não farmacológicos para alívio da dor. ${ }^{3}$ Sendo assim, o estudo traz que $39,84 \%$ das mulheres optaram pelo uso de analgesia medicamentosa. Neste quesito, há inferioridade ao apontado com taxa de uso de analgesia no trabalho de parto de 45,9\% em 2016. ${ }^{9}$ Um ponto relevante a se destacar nesse momento é a atuação da equipe de enfermagem, pois ela deve informar a mulher em trabalho de parto o que pode acontecer com a utilização da analgesia, para que ela seja capaz de tomar sua decisão de forma consciente. ${ }^{13}$

O clampeamento oportuno do cordão umbilical deverá ser realizado de Rev Enferm Atenção Saúde [Online]. Out/Dez 2021; 10(3):e202131 um a cinco minutos após o nascimento, ou quando se verificar que não existe mais sua pulsação, sendo esse um dos cuidados ao recém-nascido e sua prática deve ser executada em todos os nascimentos, exceto naqueles que possuem contraindicações relacionadas ao cordão umbilical ou quando precisa ser realizada reanimação neonatal. ${ }^{3}$ Tal prática "aumenta o nível de hemoglobina e melhora as reservas de ferro nos primeiros meses de vida". ${ }^{14}$ Desta forma, o estudo apresenta elevada taxa desta prática, sendo de $80,22 \%$ e na instituição estudada considera como clampeamento oportuno do cordão aquele que é realizado entre um e três minutos após o nascimento.

O contato pele a pele entre mãe e bebê deve ser feito logo após o nascimento e é favorável a todos os recém-nascidos, desde que eles tenham saúde e se encontrem aptos a fazê-lo na primeira hora de vida, pois auxilia na prevenção da hipotermia neonatal e incentiva o aleitamento materno, ${ }^{5}$ além de se mostrar eficaz na colonização do recém-nascido com a microbiota residente materna. ${ }^{15}$ Sendo assim, esse estudo apresenta taxa de $84,55 \%$ dos nascimentos com contato pele a pele imediatos. Esta taxa se mostra superior a do estudo explicitado anteriormente com taxa de contato pele a pele de $60,1 \%$ em $2016 .{ }^{9}$ É importante destacar que por mais que haja este contato 
entre o binômio mãe e bebê após o nascimento, na rotina da unidade estudada, tal prática não é seguida à risca, sendo este binômio separado nesta primeira hora para a realização de procedimentos de rotina, como por exemplo, medidas antropométricas e vacinação.

$\mathrm{O}$ aleitamento materno deve iniciar imediatamente após o nascimento, desde que mãe e recém-nascido estejam aptos de fazê-lo, ${ }^{5}$ pois os estudos mostram que quão antes se inicie, melhores são os resultados em relação à saúde infantil. ${ }^{16}$ Deste modo, o presente estudo apresenta uma taxa elevada de aleitamento materno após o parto, com 78,32\%. Esta variável obteve valor superior ao que apresentou taxa de estímulo a amamentação logo após o nascimento de $45 \%$ em $2016 .{ }^{9}$

O uso de ocitocina a fim de acelerar o trabalho de parto é uma prática não recomendada pela OMS. ${ }^{5}$ A recomendação é de que se faça uso somente após a confirmação de parada de progressão no primeiro período do trabalho de parto ou quando a mulher apresenta contrações uterinas que são ineficazes no segundo período do trabalho de parto. $^{3}$ Sendo assim, os achados desta pesquisa apresentam que a ocitocina foi administrada em $54,74 \%$ dos partos. Dado este inferior ao informado em pesquisa anterior que apresentou taxa de uso de ocitocina de $81,9 \%$ em $2016 .{ }^{9}$ Entretanto, outros estudos encontraram taxas mais baixas para uso da mesma de $36,4 \%$ para todas as mulheres ${ }^{11}$ e $28,6 \%{ }^{8}$ É importante ressaltar que nos registros estudados não há uma opção que sinalize a indicação do uso de ocitocina no trabalho de parto, somente se a mesma foi utilizado ou não.

A amniotomia precoce a fim de prevenir o trabalho de parto prolongado é uma prática não recomendada pela $\mathrm{OMS}^{5}$ Sua realização deve ser feita após a confirmação de parada de progressão no primeiro período do trabalho de parto ou na suspeita de prolongamento de trabalho de parto em seu segundo período. ${ }^{3}$ Os achados da pesquisa mostram que em $30,08 \%$ dos partos houve a realização de amniotomia. O resultado se difere dos demais que apresentaram taxas de realização da mesma de $21,5 \%^{8}$ e $39,1 \%$ para todas as mulheres. ${ }^{11}$ Mais uma vez, ressalta-se que nos registros estudados não há uma opção que sinalize a indicação da realização de amniotomia no trabalho de parto, somente se a mesma foi realizada ou não.

A realização de rotina da episiotomia é uma prática não preconizada no "parto vaginal espontâneo".,3 Porém, para sua realização, a mesma deve ser seletiva $^{17}$ e possuir uma justificativa. ${ }^{3}$ Dessa forma, o presente estudo apresenta $20,87 \%$ de realização desta prática. No entanto, este é inferior aos abordados na 
literatura, com taxas de episiotomia de $55 \%$ em $2016^{9}$ e $53,5 \%$ para todas as mulheres. ${ }^{11}$

Por mais que a literatura aponte que a episiotomia não deve ser um procedimento de rotina e sim de prática seletiva, os dados apresentados anteriormente mostram que sua realização ainda é difundida nos hospitais. Portanto, se faz imprescindível que o profissional que esteja assistindo o parto avalie a real necessidade da realização da mesma.

Em relação à posição de nascimento, recomenda-se "desencorajar a mulher a ficar em posição supina, decúbito dorsal horizontal, ou posição semi-supina no segundo período do trabalho de parto. A mulher deve ser incentivada a adotar qualquer outra posição que ela achar mais confortável incluindo as posições de cócoras, lateral ou quatro apoios". ${ }^{3}$ Neste estudo, $86,99 \%$ das mulheres pariram na posição litotômica, também conhecida como supina, um percentual alto e similar aos que mostraram 98,7\% em $2016^{9}$ e $91,7 \%$ para todas as mulheres. ${ }^{11}$ Sendo assim, está claro que a adoção de outros tipos de posições devem ser mais estimuladas, porém também se deve respeitar a escolha da mulher da posição de nascimento.

Quanto à manobra de Kristeller, há a recomendação de que ela "não deve ser realizada no segundo período do trabalho de parto". 3,18 Ela "desarranja a contratilidade uterina, produz hipertonia uterina, repercutindo ominosamente na vitalidade fetal; pode causar descolamento prematuro da placenta e embolia amniótica". ${ }^{14}$ Por mais que haja a recomendação da não realização desta prática, o presente estudo apresenta 2,98\% dos partos com a realização desta manobra. Este dado se mostra inferior ao apresentado em diferentes estudos com percentuais de realização da mesma de $13,6 \%$ em $2016^{9}$ e $36,1 \%$ para todas as mulheres. ${ }^{11}$ Por mais que a incidência desta prática seja menor neste estudo do que nos estudos apresentados, é importante ressaltar que, na verdade, ela deve ser completamente abolida da assistência ao parto, uma vez que pode trazer traumas físicos e risco à vida da mulher e do recémnato.

Destaca-se que a maternidade estudada faz parte dos 97 hospitais que fazem parte do projeto Apice On, o que pode ter influenciado nos resultados encontrados, além de ser uma instituição responsável pelo ensino de novos profissionais deve sempre realizar a prática baseada em evidências científicas. Cabe ressaltar, também, a atuação da enfermagem obstétrica, em parceria com a residência em saúde perinatal, que atuam na atenção ao trabalho de parto na referida instituição. 


\section{CONCLUSÃO}

O estudo aponta que na maioria dos casos, o emprego de determinadas práticas assistenciais no momento do parto foi realizado a partir de evidências científicas que são consideradas benéficas tanto para mãe, quanto para o concepto. Porém, verificou-se que uma prática considerada como proscrita, a manobra de Kristeller, ainda é realizada. Destaca-se também o quantitativo de mulheres que utilizaram ocitocina sintética no trabalho de parto e das que tiveram seus partos em posição litotômica. Esses pontos levantados estão em desacordo com as evidências científicas e precisam ser modificadas.

A pesquisa subsidiou a compreensão das práticas utilizadas na assistência ao parto normal na maternidade em questão, servindo como parâmetro norteador para a realização dos cuidados baseados em evidências científicas e na política de humanização. Acredita-se que os resultados deste estudo sirvam como catalizadores de mudanças no cenário assistencial, uma vez que eles têm o potencial de estimular tanto à equipe multiprofissional às melhores evidências científicas para a prática, quanto à população ao trazer informações pertinentes a assistência ao parto normal.

A limitação enfrentada nessa pesquisa diz respeito a falta de completude dos registros fonte da coleta dos dados, mas que de forma geral foi compensada pela amostragem ampliada.

Recomenda-se que haja monitoramento das práticas assistenciais com regularidade, uma vez que, as evidências científicas e a atenção devem manter a mesma linha de cuidado.

\section{REFERÊNCIAS}

1. Oliveira AS, Damasceno AKC, Moraes JL, Moreira KAP, Teles LMR, Gomes LFS. Tecnologias utilizadas por acompanhantes no trabalho de parto e parto: estudo descritivo. Online Braz J Nurs. [Internet]. mar 2014 [citado em 30 mar 2019]; 13(1):36-45. Disponível em: http://www.repositorio.ufc.br/bitstream/riu fc/14488/1/2014_art_asoliveira.pdf

2. Ministério da Saúde (Brasil), Secretaria de Atenção à Saúde. Portaria $\mathrm{N}^{\circ} 353$, de 14 de fevereiro de 2017. Aprova as Diretrizes Nacionais de Assistência ao Parto Normal [Internet]. Diário Oficial da União. Brasília, DF, 20 fev 2017 [citado em 19 2020]; Seção 1(36):37. Disponível em: https://www.in.gov.br/materia//asset_publisher/Kujrw0TZC2Mb/content/i d/20805342/do1-2017-02-20-portaria-n353-de-14-de-fevereiro-de-2017-20805260 3. Ministério da Saúde (Brasil), Secretaria de Ciência, Tecnologia e Insumos Estratégicos, Departamento de Gestão e Incorporação de Tecnologias em Saúde. Diretrizes Nacionais de Assistência ao Parto Normal: versão resumida [Internet]. Brasília, DF: Ministério da Saúde; 2017 [citado em 07 set 2019]. 51 p. Disponível em: http://bvsms.saude.gov.br/bvs/publica coes/diretrizes_nacionais_assistencia_parto _normal.pdf

4. Ministério da Saúde (Brasil), Secretaria de Atenção à Saúde, Departamento de Ações Programáticas e Estratégicas, 
Coordenaçãa-Geral de Saúde das

Mulheres, Coordenação-Geral de Saúde da

Criança e Aleitamento Materno. Apice on:

Aprimoramento e inovação no cuidado e

ensino em obstetrícia e neonatologia

[Internet]. Brasília, DF. Ministério da

Saúde; 2017 [citado em 2020 jan 04].

Disponível em:

http://www.cofen.gov.br/wp-

content/uploads/2017/08/Revista-Apice-

ON.pdf?undefined=undefined

5. WHO recommendations: intrapartum care for a positive childbirth experience [Internet]. Geneva: World Health Organization; 2018 [citado em 07 set 2019]. 195 p. Disponível em:

https://apps.who.int/iris/bitstream/handle/1

0665/260178/9789241550215-eng.pdf.

6. Conselho Nacional de Saúde (Brasil).

Resolução No 466, de 12 de dezembro de

2012. Aprova as diretrizes e normas

regulamentadoras de pesquisas envolvendo

seres humanos. Brasília, DF: CNS; 2012.

Disponível em:

https://conselho.saude.gov.br/resolucoes/2

012/Reso466.pdf

7. Santos JO, Pacheco TS, Oliveira OS,

Pinto VL, Gabrielloni MC, Barbieri M.

The obstetrical and newborn profile of

postpartum women in maternities in São

Paulo. Rev. Pesqui. (Univ. Fed. Estado Rio

J., Online) [Internet]. jan/mar 2015 [citado

em 07 set 2019]; 7(1):1936-45. Disponível

em:

http://www.seer.unirio.br/index.php/cuidad ofundamental/article/view/3547/pdf 1433

8. Giordano J, Surita FG. The role of the respectful maternity care model in São Paulo, Brazil: a cross-sectional study. Birth [Internet]. ago 2019 [citado em 28 out 2019]; 46(3): 509-16.

doi:https://doi.org/10.1111/birt.12448

9. Lopes GC, Gonçalves AC, Gouveia HG, Armellini CJ. Atenção ao parto e nascimento em hospital universitário:

comparação de práticas desenvolvidas após Rede Cegonha. Rev Latinoam

Enferm. [Internet]. 2019 [citado em 07 set 2019 ]; 27:e3139. Disponível em: http://www.scielo.br/scielo.php?script=sci

_arttext\&pid=S0104-

$11692019000100327 \& \operatorname{lng}=$ en

10. Presidência da República (Brasil). Lei

Federal n ${ }^{\circ} 11.108$, de 07 de abril de 2005.

Altera a Lei 8.080 de 19 de setembro de

1990 para garantir as parturientes o direito

à presença de acompanhante durante $o$

trabalho de parto, parto e pós-parto

imediato, no âmbito do Sistema Único de

Saúde - SUS [Internet]. Diário Oficial da

União, Brasília, 08 abr 2005 [citado em 07 set 2019]. Disponível em:

http://www.planalto.gov.br/ccivil_03/_Ato

2004-2006/2005/Lei/L11108.htm

11. Leal MC, Pereira APE, Domingues

RMSM, Theme Filha MM, Dias MAB,

Nakamura-Pereira M, et al. Intervenções obstétricas durante o trabalho de parto e parto em mulheres brasileiras de risco habitual. Cad Saúde Pública [Internet]. 2014 ago [citado em 07 set 2019]; 30(Supl 1):17-32. Disponível em:

http://www.scielo.br/scielo.php?script=sci

_arttext\&pid=S0102-311X2014001300

$005 \& \operatorname{lng}=\mathrm{pt}$

12. Santos AHL, Nicácio MC, Pereira

ALF, Oliveira TCM, Progianti JM.

Práticas de assistência ao parto normal: formação na modalidade de residência.

Rev Enferm UFPE on line [Internet]. jan 2017 [citado em 08 set 2019]; 11(1):1-9.

Disponível em:

https://periodicos.ufpe.br/revistas/revistaen fermagem/article/view/11871/14319 13. Fernandes RLV, Damasceno AKC, Herculano MMS, Martins RST, Oriá MOB. Analgesia obstétrica farmacológica: um estudo sobre os desfechos obstétricos e neonatais. Rev Rene [Internet]. set/out 2017 [citado em 08 set 2019]; 18(5):68794. Disponível em:

http://periodicos.ufc.br/rene/article/view/3 0847/71507

14. Rezende Filho J, Montenegro CAB.

Obstetrícia. 13ed. Rio de Janeiro:

Guanabara Koogan; 2016.

15. Sociedade Brasileira de Pediatria, Departamento Científico de Neonatologia. Nascimento seguro [Internet]. Rio de 
Janeiro: Sociedade Brasileira de Pediatria; abr 2018 [citado em 20 set 2019]. 16 p. (Documento Científico; n. 3). Disponível em:

https://www.sbp.com.br/fileadmin/user_up load/Neonatologia_-_20880b-DC_-

_Nascimento_seguro__003_.pdf

16. Rocha LB, Araujo FMS, Rocha NCO, Almeida CD, Santos MO, Rocha CHR.

Aleitamento materno na primeira hora de vida: uma revisão de literatura. Rev Med Saúde Brasília [Internet]. 2017 [citado em 20 set 2019]; 6(3):384-94. Disponível em: https://portalrevistas.ucb.br/index.php/rms br/article/view/8318/5490

17. Federação Brasileira das Associações de Ginecologia e Obstetrícia.

Recomendações Febrasgo parte II -

Episiotomia [Internet]. São Paulo:

FEBRASGO; dez 2018 [citado em 07 abr 2019]. Disponível em:

RECEBIDO: $27 / 05 / 2020$

APROVADO: $01 / 12 / 2020$

PUBLICADO: dez/2021 https://www.febrasgo.org.br/pt/noticias/ite $\mathrm{m} / 715$-recomendacoes-febrasgo-parte-iiepisiotomia\#

18. Ministério da Saúde (Brasil), Comissão Nacional de Incorporação de Tecnologias do SUS, Secretaria de Ciência, Tecnologia e Insumos Estratégicos. Diretriz nacional de assistência ao parto normal: relatório de recomendação [Internet]. Brasília, DF: Ministério da Saúde; 2016 [citado em 08 set 2019]. 381 p. Disponível em: http://conitec.gov.br/images/Consultas/201 6/Relatorio

_Diretriz-PartoNormal_CP.pdf 